\title{
Emotion Recognition Using Ensemble Bagged Tree Classifier and Electroencephalogram Signals
}

\author{
Olori Emmanuel Obaro ${ }^{1}$, Benjamin Segun Aribisala ${ }^{1,2}$ and Patrick Adeomo Owate ${ }^{1}$
}

\begin{abstract}
${ }^{1}$ Department of Computer Science, Faculty of Science, Lagos State University, Nigeria

${ }^{2}$ Department of Neuroimaging Sciences, Center for Clinical Brain Sciences, University of Edinburgh, Edinburgh UK
\end{abstract}

\begin{abstract}
:
Introduction: Emotion plays a key role in our daily life and work, especially in decision making, as people's moods can influence their mode of communication, behaviour or productivity. Emotion recognition has attracted some research works and medical imaging technology offers tools for emotion classification.

Aims: The aim of this work is to develop a machine leaning technique for recognizing emotion based on Electroencephalogram (EEG) data

Materials and Methods: Experimentation was based on a publicly available EEG Dataset for Emotion Analysis using Physiological (DEAP). The data comprises of EEG signals acquired from thirty two adults while watching forty different musical video clips of one minute each. Participants rated each video in terms of four emotional states, namely, arousal, valence, like/dislike and dominance. We extracted three features from the dataset, these are wavelet energy, wavelet entropy and standard deviation. We then classified the extracted features into four emotional states, namely, High Valence/High Arousal, High Valance/Low Arousal, Low Valence/High Arousal, and Low Valence/Low Arousal using Ensemble Bagged Trees. Support Vector Machine was also used for classification just for comparison.

Results: Ensemble Bagged Trees gave sensitivity, specificity and accuracy of $97.54 \%, 99.21 \%$ and $97.80 \%$ respectively. Support Vector Machine and Ensemble Boosted Tree gave similar results.

Conclusion: Our results showed that machine learning classification of emotion using EEG data is very promising. This can be useful in management and treatment of patients with emotional related problems, especially those with expression problems like Amyotrophic Lateral Sclerosis.

Keywords: Electroencephalogram, Emotions Recognition, Ensemble Classification, Ensemble Bagged Trees, Machine Learning
\end{abstract}




\subsection{INTRODUCTION}

\subsection{EMOTION}

Emotion can be described as a strong feeling derived from one's circumstances, mood, or relationships with others. Emotion is important as it plays a key role in our daily life and work, especially in decision making. Individual's emotion can greatly influence their mode of communication, as well as their behaviour and productivity. Emotion can sometimes manifest as facial expression, voice or physical behaviourr but sometimes, emotion may not be visible to observers. The brain is the source of emotions, as it controls the actual emotion of a particular person. In view of this, emotion state is commonly measured by capturing brain activities. Emotion can be positive or negative[1]. Positive emotions are those emotions that do not contain any bad symptoms such as pain, anger or discomfort, while negative emotions are those that manifests as bad signs such as anger, frustration, guilt, nervousness and fear.

Emotions can be classified into four quadrants namely, High Valence/High Arousal (HVHA), High Valance/Low Arousal (HVLA), Low Valence/High Arousal (LVHA), and Low Valence/Low Arousal (LVLA). This emotion classification approach uses the arousal-valance scale which was first proposed by Russell[2], and it has been widely accepted for classification of emotions. The concept is that each emotional state can be placed on a two-dimensional plane with arousal and valence as the axes. Arousal can range from inactive (e.g. uninterested, bored) to active (e.g. alert, excited), whereas valence ranges from unpleasant (e.g. sad, stressed) to pleasant (e.g. happy, elated).

Emotions are known to generate brain signals which can be captured using different measuring techniques such as Near Infra-Red Spectroscopy (NIRS), functional Magnetic Resonance Imaging (fMRI), Magneto Encephalography (MEG) and Electroencephalography (EEG). However, due to some limitations of NIRS, fMRI and MEG, EEG has been proposed to be a preferred emotion imaging technique based on its high temporal resolution at a level of milliseconds and also a non-invasive technique [3]. In recent years, emotion recognition from EEG has gained some LASU Journal of Research and Review in Science attention [4-8]. Emotion detection has many application areas. For example it is useful in management and treatment of emotional related conditions, especially those with expression problems like Amyotrophic Lateral Sclerosis. It also plays a good role in Brain Computer Interface (BCI) systems as it helps to develop techniques that can improve the communication between humans and machines [9-11].

\subsection{MACHINE CLASSIFICATION}

Emotion was conventionally classified using manual methods, but the advent of computer technology has led to the use of computerized methods of classifying emotion. A machine learning technique called Support Vector Machine (SVM) has been previously used for classification of EEG data and has also provided good results[12], however with limited accuracy[13, 14], especially in multiclass problems. The main focus of the research is the need to improve the classification of emotion. Improved classification could lead to improved diagnosis of emotion related problem and could also increase the application areas of emotion recognition. Here we explore the use of Ensemble bagged tree and Ensemble boosted tree for classification of EEG data.

Ensembles combines several decision trees to produce a better predictive performance, such that a group of weak learners come together to form a strong learner[15]. Bagging also called Bootstrap Aggregation, works in such a way that it creates several subset of data from the training sample chosen randomly, each collection of subset data is used to train their decision trees, which ends up with an ensemble of different models. The average of all the predictions from different trees are used which is more robust than a single decision tree,

The performance metrics used in this research are sensitivity, specificity, precision, accuracy and Area under the Curve (AUC). Using binary classification as a case study, sensitivity also called True Positive Rate (TPR) or recall 
measures the proportion of actual positive that are correctly identified as such. Specificity also called True Negative Rate (TNR) measures the actual negative that are correctly identified as such. Precision or positive predictive value (PPV) can be defined as the proportion of the true positives against all positive results (both true positives and false positives). AUC measures an estimate of how well a classifier model can predict a random sample (where actual label is the positive instance) as a positive instance in comparison to predicting a random sample (where actual label is the negative instance) as a negative instance.

Also, accuracy is the overall percentage of correctly classified instances. TPR, TNR, Precision and accuracy range between 0 and 100 where 0 is the worst and 100 is the best. FPR and FNR also range between 0 and 100 but 0 is the best and 100 is the worst. AUC ranges between 0 and 1 with 0 being the worst and 1 being the best.

\subsection{MATERIALS AND METHODS}

The dataset used for this research was obtained from a publicly available database (https://www.eecs.qmul.ac.uk/mmv/datasets/deap/ ) called the Database for Emotion Analysis using Physiological signals (DEAP)[16]. We extracted some features using Discrete Wavelet Transforms on the pre-processed dataset with the 32 EEG channels. Afterward, three classification algorithms was used to classify the signals based on Russell's scale[2].

\subsection{DATA DESCRIPTION}

The full detail of the experiment carried out during data acquisition is available elsewhere[16]. In brief, music video clips were used as visual stimuli to elicit different emotional status. For each video, a one-minute highlight was selected automatically[16]. 32 participants watched 40 one-minute musical videos each, as they watched the videos, the EEG and peripheral signal were recorded.

The experiments were performed in two laboratory environments with controlled illumination. EEG and peripheral physiological signals were recorded using a Biosemi ActiveTwo system on a dedicated recording PC. EEG was recorded at a sampling rate of $512 \mathrm{~Hz}$ using 32 active $\mathrm{AgCl}$ electrodes (placed according to the international $10-20$ system). The 32 healthy participants which involved equal numbers of male and female of age between 19 and 37 with a mean age of 26.9, the participants were presented with a trial video before watching the one-minute video. The 40 one-minute videos were selected in such a way that one belongs to valence-arousal space (HVHA, HVLA, LVHA, and LVLA) proposed by James Russell[2].

Each participant rated the video in terms of arousal, valence, like/dislike and dominance on scale of 1-9, 1 for low and 9 for high scale.

\subsection{PRE-PROCESSING}

The data used for this research was pre-processed as described in DEAP[16]. The pre-processing can be summarized as follows. The raw data was preprocessed by resampling to $128 \mathrm{~Hz}$. The signals were passed through the band-pass filter which was set to $4 \mathrm{~Hz}$ on the minimum and $45 \mathrm{~Hz}$ as maximum to obtain frequency related cognitive signals in EEG, such as the frequency band of theta $(4-8 \mathrm{~Hz})$, frequency band of alpha $(8-12$ $\mathrm{Hz})$ and frequency band of beta $(8-40 \mathrm{~Hz})$, Electrooculography (EOG) artifacts such as eye movements was also removed. The data was segmented into 60 seconds trial and a 3 second pre-trial baseline removed.

The experiment used 40 video clips and 40 channel (32 EEG channel and 8 peripheral physiological channel) to extract 8064 EEG/physiological signal data from each channel, the 8064 represent EEG values recorded over the duration of 1 minute of the participants watching the video. Each participant file is arranged as a 3D data i.e. $40 \times 40 \times 8064$, which represents the video/trial $\mathrm{x}$ channel $\mathrm{x}$ EEG values.

\subsection{FEATURE EXTRACTION}

EEG signals are highly non-stationary and hence time-frequency domain methods e.g. Discrete Wavelet Transforms (DWT) have been suggested to be good for feature extraction[17, 18].

Since the focus of this research is on EEG signals, we were able to extract three features from the 32 EEG electrodes used out of the 40 channels consisting of 32 EEG and 8 peripheral physiological channel, the features extracted were energy of the signals in form of wavelet energy, 
the degree of order/disorder of the signal in form of wavelet entropy and a statistical feature i.e. the standard deviation, alongside were their arousal and valence ratings. To extract the features correctly, the wave signal of one minute was divided into 6 segments of 6 seconds, thus giving 10 total of segments where the three features was extracted from each of the segments.

\subsection{CLASSIFICATION}

After extracting the three features from each channel alongside with the arousal and valence ratings, participant number, videos and wave segments, the three features from each channel was combined together which gave a total of 101 features. . Since the rating of the videos were based on arousal and valence, a scale of 1-9 was used with 1 being the lowest and 9 being the highest for each rating. Any rating of 5 and abovewas taken as high and any rating of 5 and below was taken as low. Similarly, the participant rating with 5 and above for valance and 5 and above for arousal is tagged as HVHA, rating of 5 and above for valance and 5 and below for arousal is tagged as HVLA, rating of 5 and belowfor valance and rating of 5 and above for arousal is tagged as LVHA, lastly, rating of 5 and below for valance and rating of 5 and below for arousal is tagged as LVLA.

Once each class has been identified, the sample data was randomly partitioned into training set, validation set and test set using $\mathrm{k}$-fold cross validation (where $\mathrm{k}=10$ ), each set has a total of 1280 dataset.

Ensemble bagged tree and Ensemble boosted tree were implemented in MATLAB and used for the classification. Thirty decision trees were used in the ensemble bagged tree[19] experiment. The 30 decision trees were learnt sequentially with early learners fitting simple models to the data and then analyzing the data for errors. That is, it fits consecutive trees and at every step, the goal is to solve for net error from the prior tree. SVM was also implemented for comparison. To measure the performance for each classification algorithm, a confusion matrix was generated to extract necessary measures for each classifier such sensitivity (TPR), specificity (TNR), Area under the curve and accuracy.

\section{RESULTS}

Table 1 shows the average performance of the Ensemble Bagged Tree and Ensemble Boosted Tree, SVM was included for comparison. The average of all classes using Ensemble Bagged Tree for precision, specificity, sensitivity and average accuracy is $97.97 \%, 99.21 \%, 97.54$ and $97.80 \%$ respectively. While the average of all classes using Ensemble Boosted Tree for precision, specificity, sensitivity and average accuracy is $97.97 \%, 99.19 \%, 97.50 \%$ and $97.70 \%$ respectively. Lastly, the average of all classes using SVM for precision, specificity, sensitivity and average accuracy is $97.76 \%, 97.58 \%, 97.35 \%$ and $97.50 \%$ respectively. Figure 1, shows a bar chart plotting sensitivity, specificity and precision for the three classifiers.

Table 1: Performance of the Proposed Classifiers Using Precision, Specificity, Sensitivity and Accuracy Measures

\begin{tabular}{|l|l|l|l|l|}
\hline Classifier & $\begin{array}{l}\text { Precision } \\
(\%)\end{array}$ & $\begin{array}{l}\text { Specificity } \\
(\%)\end{array}$ & $\begin{array}{l}\text { Sensitivity } \\
(\%)\end{array}$ & $\begin{array}{l}\text { AVERAGE } \\
\text { ACURACY } \\
(\%)\end{array}$ \\
\hline $\begin{array}{l}\text { Ensemble } \\
\text { Bagged } \\
\text { Tree }\end{array}$ & $\mathbf{9 7 . 9 7}$ & $\mathbf{9 9 . 2 1}$ & $\mathbf{9 7 . 5 4}$ & $\mathbf{9 7 . 8 0}$ \\
\hline $\begin{array}{l}\text { Ensemble } \\
\text { Boosted } \\
\text { Tree }\end{array}$ & 97.97 & $\mathbf{9 9 . 1 9}$ & $\mathbf{9 7 . 5 0}$ & $\mathbf{9 7 . 7 0}$ \\
\hline $\begin{array}{l}\text { Support } \\
\text { Vector } \\
\text { Machine }\end{array}$ & $\mathbf{9 7 . 7 6}$ & $\mathbf{9 7 . 5 8}$ & 97.35 & $\mathbf{9 7 . 5 0}$ \\
\hline
\end{tabular}

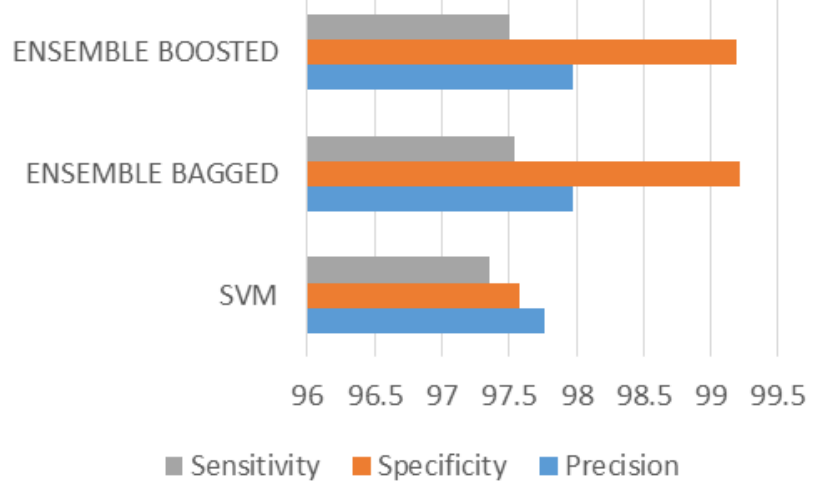

Figure 1: Bar Charts showing the overall performance measure for each of the Classification Algorithms 


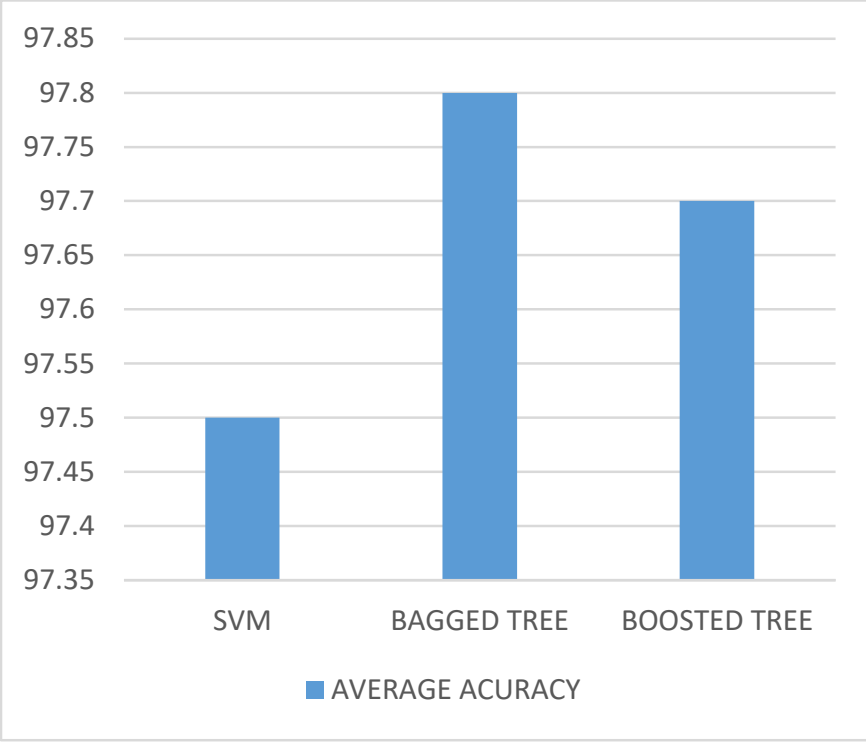

Figure 2: Charts showing overall average performance of the three classification Algorithm

Figure 2 shows the average accuracy for the three classifiers. The accurate accuracy were $97.80 \%$, 97.70\% and $97.50 \%$ for Ensemble Bagged Tree, Ensemble Boosted Tree and SVM respectively. These show that both Ensembles algorithm performed slightly better than SVM. Also, Ensemble Bagged Tree performed slightly better than Ensemble Boosted as both have the same precision, the same sensitivity but the specificity of Ensemble Bagged Trees is higher than that of Ensemble Boosted Trees

\section{CONCLUSION}

This research developed a machine learning technique that can recognize emotion based on Ensemble classification technique using Electroencephalogram data. A preprocessed dataset of Emotion was obtained from a publicly available Dataset for Emotion Analysis using Physiological, DEAP, where Discrete Wavelet Transforms was used to extract features such as wavelet energy, wavelet entropy and standard deviation. We then classified the extracted features into four emotional states, namely, High Valence/High Arousal, High Valance/Low Arousal, Low Valence/High Arousal, and Low Valence/Low Arousal using Ensemble Bagged Trees and Ensemble Boosted Trees.

We assessed performance of Ensemble Bagged Tree using sensitivity analysis and also by comparing it with a common classifier, called the Support Vector Machine.

Our results showed that machine learning classification of emotion using EEG data is very promising and that Ensemble Bagged Trees gave the best result in comparison with other two classifiers investigated. The proposed classifier i.e. the Ensemble Bagged Trees can help in the management and treatment of patients, with emotion related problems e.g. those with expression problems like Amyotrophic Lateral Sclerosis.

\section{ACKNOWLEDGEMENTS}

The authors appreciate Mr. Mayowa Adeyemi and Mr. Bamiro Ustaz of the Department of Computer Science, Lagos State University for their help in data collation.

\section{COMPETING INTERESTS}

Authors have declared that no competing interests exist.

\section{AUTHORS' CONTRIBUTIONS}

Author BSA designed the study, supervised literature review, supervised data analysis and wrote the final draft the paper. Author OEO obtained and analyzed data, conducted literature review, wrote the first draft of the paper and performed all experiments. Author PAO conducted literature review, read and approved the final manuscript.

\section{REFERENCES}

1. Solomon, Robert C. Emotion. 2017 12-15-2017; All about Emotion, Page visited on: 6/02/2018 Url: https://www.britannica.com/topic/emotion

2. Russell, James A, A circumplex model of affect. Journal of personality and social psychology, 1980. 39(6): p. 1161.

3. Devi, Vidya and G Thippeswamy, A survey on signal acquistion and feature extraction techniques. International Journal of Computer Applications. 975: p. 8887. 
4. Al-Fahoum, Amjed S and Ausilah A Al-Fraihat, Methods of eeg signal features extraction using linear analysis in frequency and time-frequency domains. ISRN neuroscience, 2014. 2014.

5. Ali, Mouhannad, Ahmad Haj Mosa, Fadi Al Machot, and Kyandoghere Kyamakya. Eegbased emotion recognition approach for $e^{-}$ healthcare applications. in Ubiquitous and Future Networks (ICUFN), 2016 Eighth International Conference on. 2016. IEEE.

6. Bos, Danny Oude, Eeg-based emotion recognition. The Influence of Visual and Auditory Stimuli, 2006. 56(3): p. 1-17.

7. El-Kafrawy, Noran M, Doaa Hegazy, and Mohamed Fahmy Tolba. Features extraction and classification of eeg signals using empirical mode decomposition and support vector machine. in International Conference on Advanced Machine Learning Technologies and Applications. 2014. Springer.

8. Hjelm, Sara Ilstedt and Carolina Browall. Brainball-using brain activity for cool competition. in Proceedings of NordiCHI. 2000.

9. Machado, Sergio, Fernanda Araújo, Flávia Paes, Bruna Velasques, Mario Cunha, Henning Budde, Luis F Basile, Renato Anghinah, Oscar AriasCarrión, and Mauricio Cagy, Eeg-based braincomputer interfaces: An overview of basic concepts and clinical applications in neurorehabilitation. Reviews in the Neurosciences, 2010. 21(6): p. 451-468.

10. Wang, Chaunchu, Cuntai Guan, and Haihong Zhang. P300 brain-computer interface design for communication and control applications. in Engineering in Medicine and Biology Society, 2005. IEEE-EMBS 2005. 27th Annual International Conference of the. 2006. IEEE.

11. Felzer, Torsten, Rudolf Fischer, Thomas Groensfelder, and Rainer Nordmann. Alternative control system for operating a pc using intentional muscle contractions only. in OnlineProc. CSUN Conf. 2005.
12. Hosni, Sarah M, Mahmoud E Gadallah, Sayed F Bahgat, and Mohamed S AbdelWahab. Classification of eeg signals using different feature extraction techniques for mental-task bci. in Computer Engineering \& Systems, 2007. ICCES'07. International Conference on. 2007. IEEE.

13. Prajapati, Gend Lal and Arti Patle. On performing classification using svm with radial basis and polynomial kernel functions. in 2010 3rd International Conference on Emerging Trends in Engineering and Technology. 2010. IEEE.

14. Jirayucharoensak, Suwicha, Setha Pan-Ngum, and Pasin Israsena, Eeg-based emotion recognition using deep learning network with principal component based covariate shift adaptation. The Scientific World Journal, 2014. 2014.

15. Bühlmann, P, Handbook of computational statistics: Concepts and methods, chapter bagging, boosting and ensemble methods. 2010, Springer.

16. Koelstra, Sander, Christian Muhl, Mohammad Soleymani, Jong-Seok Lee, Ashkan Yazdani, Touradj Ebrahimi, Thierry Pun, Anton Nijholt, and Ioannis Patras, Deap: A database for emotion analysis; using physiological signals. IEEE Transactions on Affective Computing, 2012. 3(1): p. 18-31.

17. Samar, Vincent J, Ajit Bopardikar, Raghuveer Rao, and Kenneth Swartz, Wavelet analysis of neuroelectric waveforms: A conceptual tutorial. Brain and language, 1999. 66(1): p. 7-60.

18. Subasi, Abdulhamit, Eeg signal classification using wavelet feature extraction and a mixture of expert model. Expert Systems with Applications, 2007. 32(4): p. 1084-1093.

19. Breiman, Leo, Bagging predictors. Machine learning, 1996. 24(2): p. 123-140. 